\title{
RoTFL1c of Rosa multiflora has a dual-function in suppressing reproductive growth and promoting vegetative growth of Arabidopsis
}

\author{
Fang Wang ${ }^{1 \dagger}$, Lijuan $\operatorname{Lian}^{1 \dagger}$, Yunfeng Liu ${ }^{1}$, Yuman Zhang ${ }^{2}$, Rongxiang Fang ${ }^{2 *}$ \\ \& Qinglin Liu ${ }^{*}$ \\ ${ }^{1}$ Department of Ornamental Horticulture and Landscape Architecture, China Agriculture University, Beijing 100193, China; \\ ${ }^{2}$ State Key Laboratory of Plant Genomics, Institute of Microbiology, Chinese Academy of Sciences, Beijing 100101, China
}

Citation: Wang, F., Lian, L., Liu, Y., Zhang, Y., Fang, R., and Liu, Q. (2016). RoTFL1c of Rosa multiflora has a dual-function in suppressing reproductive growth and promoting vegetative growth of Arabidopsis. Sci China Life Sci 61, 1599-1601. doi: 10.1007/s11427-016-0082-1

Dear Editor,

TFL1 homologues play a key role in the flowering habit of roses. To analyze the function of the newly isolated TFL1 gene of Rosa multiflora (Chen et al., 2013), named RoTFL1c, we performed overexpression analyses in Arabidopsis thaliana. When compared with empty vector control and wild type Arabidopsis plants, RoTFL1c overexpressing transgenic plants exhibited strong phenotypes such as a clustered habit, an increased number of rosette leaves, late flowering or failure to flower. These phenotypes were basically consistent with, but more resilient than, those observed with overexpression of TFLI or its Rosaceae homologous genes in Arabidopsis. We concluded that the rose RoTFL1c gene not only plays important roles in delaying and/or suppressing flowering, but also in promoting vegetative growth of the plant.

The cDNA of RoTFL1c was cloned from R. multiflora “Albo-plena" by RT-PCR with specific primers (Table S1 and Figure S1 in Supporting Information). We compared the amino acid sequence of RoTFL1c with those of TFL1 homologues. The AA sequence identities between RoTFL1c and other TFL1 homologues varied depending on their tax-

†Contributed equally to this work

*Corresponding authors (Rongxiang Fang, email: fangrx@im.ac.cn;

Qinglin Liu, email: liuq1@cau.edu.cn) onomic relations. The AA identities were $76 \%$ with TFL1 of A. thaliana and $87 \%$ or more when compared with other plants in Rosaceae. The highest identity in the rose family was $99 \%$ with RoKSN of Rosa chinensis var. spontanea (Figure S2 in Supporting Information). This is consistent with the high identities of homologous sequences of TFL1 in Rosaceae (Iwata et al., 2012).

To overexpress RoTFL1c in Arabidopsis, the ORF fragment of RoTFL1c was amplified by PCR with primers (Table S1 in Supporting Information), double-digested with $X b a$ I and $S a c$ I, and cloned into the plant expression vector pBI121 under the direction of the CaMV 35S promoter (RoTFL1c-OE) (Figure S3 in Supporting Information). The pBI121-RoTFL1c (RoTFL1c-OE) and pBI121 plasmids were separately introduced into Agrobacterium tumefaciens LBA4404 by electroporation. Arabidopsis was transformed using the floral-dip method. The resulting transgenic seeds of the first generation $\left(\mathrm{T}_{0}\right)$ were screened on Murashige \& Skoog (MS) medium containing $80 \mathrm{mg} \mathrm{L}^{-1}$ of kanamycin. In three replicate screenings, 11,19 and 12 kanamycin-resistant seedlings were obtained. These kanamycin-resistant plants were checked for the presence of the RTFLc sequence by PCR and 9, 17 and 13 plants were positive (Figure 1A). In comparison with wild type and pBI121 vector-transformed plants, the phenotypes of RoTFL1c-OE plants could be categorized into four classes. Class I plants showed a cluster habit and Class II plants had a significant 

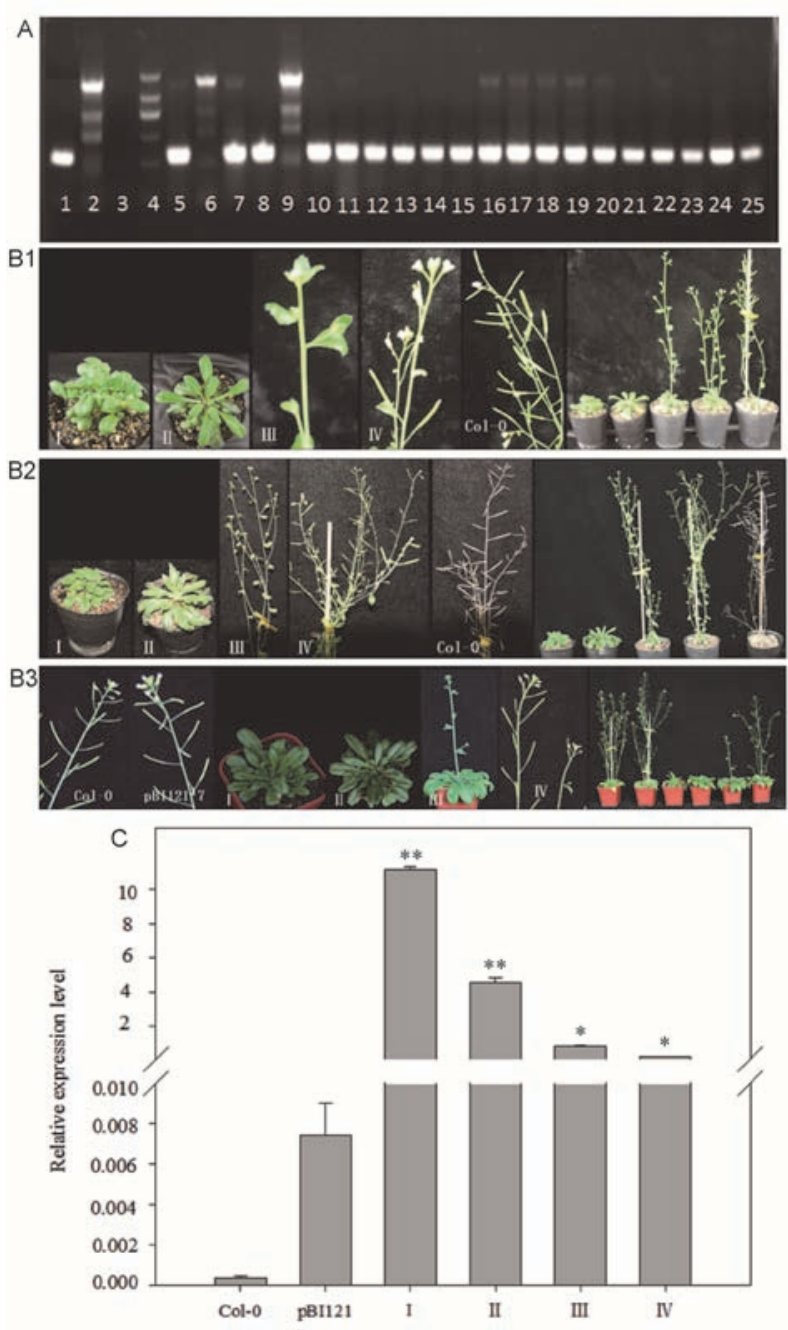

Figure 1 (Color online) Molecular detection and phenotype analysis of RoTFL1c-OE transgenic Arabidopsis thaliana. A, Molecular detection of transgenic plants. From left to right: 1, positive control; 2, wild type; 3, blank control; 4, DNA ladder marker II; 5-25, transgenic plants. B1, B2, and $\mathrm{B} 3$ indicate the different batches of RoTFL1c-OE transgenic plants that either showed a delay in blossoming or a failure to blossom. Classes I, II, III and IV indicate different types of phenotypes observed in the RoTFL1c-OE transgenic plants. Class I had clustered branches, Class II had a significant number of rosette leaves, Class III had no blossoms, and Class IV showed delayed flowering. Col-0 is the wild type and PBI121 is the A. thaliana plant transformed with the pBI121 empty vector. C, A comparison of the expression levels in RoTFL1c-OE transgenic plants with different phenotypes. Error bars indicate SE $(n=3)$. * and ** indicate significant difference at $P \leqslant 0.05$ and $P \leqslant 0.01$, respectively, in an SPSS $t$-test.

increase in the number of rosettes. Neither class bolted after a 4-month growth period. The flower buds of Class III plants failed to open at both the top of the stem and its lateral branches, or aborted after bloom, although there was an increased number of branches. Class IV plants, although they had normal flowers, showed delayed flowering (Figure 1B). All four classes showed similar results in all three batches (Table S2 in Supporting Information).

The expression level of RoTFLlc in transgenic plants and wild type Arabidopsis was analyzed using real-time
PCR. The results showed that the expression level of RoTFL1c in Class III, which did not flower, was clearly higher than that in Class IV (Figure 1C), while the expression levels in Classes II and I were even higher than those in Classes III and IV. The results showed that the expression level of RoTFL1c was negatively associated with flowering.

There were four classes of phenotypes in the transgenic plants overexpressing RoTFL1c. These classes were closely related with the expression level of RoTFL1c. With increased levels of RoTFL1c expression from Class IV to Class I, the reproductive growth of transgenic plants was increasingly suppressed or vegetative growth was promoted. Such strong variations as a cluster habit (Class I) or an increase in rosettes without shoots (Class II) have not been seen before in transgenic A. thaliana carrying TFL1 homologues. The phenotypes of Class III were similar to those of A. thaliana overexpressing TFL1 homologues from Cornus officinalis and Saccharum officinarum. The phenotypes of Class IV were similar to those found in A. thaliana overexpressing TFL1 homologues from Prunus mume, Prunus persica and Prunus serotina. They were also similar to those in tfl1-1 mutant A. thaliana overexpressing TFL1 homologues from Malus domestica and R. chinensis var. spontanea in (Kotoda and Wada, 2005; Randoux et al., 2014), which restored the mutation.

The function of TFL1 was found to be different between annuals and perennials. For example, mutation of FvTFL1 caused recurrent flowering in Fragaria vesca. The loss of function of TFL1 homologues from apple and pear by antisense technology resulted in recurrent flowering in transgenic plants. The reason for the nonexpression of $R o K S N$ in continuously flowering roses was found to be a retrotransposon insertion (Wang et al., 2012). However, overexpression of RoKSN in continuously flowering roses resulted in no flowering at all. Although the function of TFL1 is universal in Rosaceae as a flowering inhibitor, the corresponding phenotypes (including continuous flowering, recurrent blooming, flowering once, and no flowering types), may be caused by different expression levels of TFL1 homologues or by other factors associated with the flowering habits of roses.

Compliance and ethics The author(s) declare that they have no conflict of interest.

Acknowledgements This work was supported by the National High-tech $R \& D$ Program (2011AA100208), and the State Key Laboratory of Plant Genomics, Institute of Microbiology, Chinese Academy of Sciences (2016A0219-24).

Chen, Y.H., Jiang, P., Thammannagowda, S., Liang, H.Y., and Wilde, H.D. (2013). Characterization of peach TFLI and comparison with FT/TFL1 gene families of the Rosaceae. J Am Soc Hortic Sci 138, 12-17.

Iwata, H., Gaston, A., Remay, A., Thouroude, T., Jeauffre, J., Kawamura, 
K., Oyant, L.H., Araki, T., Denoyes, B., and Foucher, F. (2012). The TFL1 homologue KSN is a regulator of continuous flowering in rose and strawberry. Plant J 69, 116-125.

Kotoda, N., and Wada, M. (2005). MdTFL1, a TFL1-like gene of apple, retards the transition from the vegetative to reproductive phase in transgenic Arabidopsis. Plant Sci 168, 95-104.

Randoux, M., Daviere, J.M., Jeauffre, J.,Thouroude, T., Pierre, S., Toual- bia, Y., Perrotte, J., Reynoird, J.P., Jammes, M.J., Oyant, L.H., and Foucher, F. (2014). RoKSN, a floral repressor, forms protein complexes with RoFD and RoFT to regulate vegetative and reproductive development in rose. New Phytol 202, 161-173.

Wang, L.N., Liu, Y.F, Zhang, Y.M, Fang, R.X., and Liu Q.L. (2012). The expression level of Rosa Terminal Flower 1 (RTFL1) is related with recurrent flowering in roses. Mol Biol Rep 39, 3737-3746.

Open Access This article is distributed under the terms of the Creative Commons Attribution License which permits any use, distribution, and reproduction in any medium, provided the original author(s) and source are credited.

\section{SUPPORTING INFORMATION}

Figure S1 Electrophoresis analysis of the RoTFL1c ORF fragment.

Figure S2 Sequence alignment (A) and phylogenic tree (B) of TFL1 protein homologues in Rosaceae and Arabidopsis thaliana.

Figure S3 Sketch map of the plant overexpression vector pBI121-RoTFL1c.

Table S1 Primers used in this study

Table S2 Statistics of RoTFL1c-OE transgenic Arabidopsis thaliana with various phenotypes

The supporting information is available online at http://life.scichina.com and https://link.springer.com. The supporting materials are published as submitted, without typesetting or editing. The responsibility for scientific accuracy and content remains entirely with the authors. 